\title{
Ruptured Sinus of Valsalva Aneurysm Complicating Infective Endocarditis: A Case Report
}

\author{
HAQUE HF ${ }^{\mathrm{a}}$, AMIN MG ${ }^{\mathrm{b}}$, MUSA AKM ${ }^{\mathrm{c}}, \mathrm{AHMED} \mathrm{AKMS}^{\mathrm{d}}$, RAHIM MA ${ }^{\mathrm{e}}, \mathrm{AFROZF}^{\mathrm{a}}$, \\ AFROZE SR $^{\mathrm{a}}$, DATTA ${ }^{f}$
}

\begin{abstract}
:
Sinus of Valsalva aneurysm (SVA) is an uncommon cardiac anomaly, usually congenital but may be acquired \& rarely ruptures. A 24-year-old male presented with a history of prolonged fever, loss of appetite and significant weight loss along with exertional dyspnoea, palpitation and mild chest pain together with clubbed fingers and toes, mild splenomegaly and a continuous machinery murmur over the left sternal edge with associated thrill. Transthoracic
\end{abstract}

echocardiogram revealed ruptured sinus of Valsalva aneurysm with shunt from aorta to right atrium $(R A)$ and vegetation. He was diagnosed as infective endocarditis with rupture of sinus of Valsalva aneurysm and treated with antibiotics and referred to the cardiothoracic surgeon for repair of ruptured SVA.

Key words: Sinus of Valsalva aneurysm, infective endocarditis.

(Birdem Med J 2015; 5(Supplement 1): 52-55)

\section{Introduction:}

Sinus of Valsalva is a localized bulging in the aortic root opposite to the cusps of the aortic valve. Aneurysm of the sinus of Valsalva (SVA) is a rare condition which is usually a congenital cardiac anomaly but it can also be secondary to chest trauma, infection involving aortic root, syphilis, Behçet disease, atherosclerosis and Marfan syndrome. ${ }^{1-5}$ SVA is usually clinically silent but may vary from a mild, asymptomatic dilatation detected in routine 2-dimensional echocardiography to symptomatic presentations related to the compression

a. Dr. Hasna Fahmima Haque, FCPS (Medicine), Farhana Afroz, FCPS (Medicine), Samira Rahat Afroze FCPS (Medicine), Registrar, Department of Medicine, BIRDEM General Hospital \& Ibrahim Medical College.

b. Dr. Gaffar Amin, MD (Cardiology), Junior Consultant, Department of Cardiology, Dhaka Medical College.

c. Prof. AKM Musa, Professor \& Head of the Department of Medicine, BIRDEM General Hospital \& Ibrahim Medical College.

d. Dr. AKM Shaheen Ahmed, FCPS (Medicine), Associate Professor, Department of Medicine, BIRDEM General Hospital \& Ibrahim Medical College.

e. Dr. Muhammad Abdur Rahim, FCPS (Medicine), Assistant Professor, Department of Nephrology, BIRDEM General Hospital \& Ibrahim Medical College.

f. Dr. Nandini Datta, Resident Medical Officer, Department of Nephrology, BIRDEM General Hospital.

Address of Correspondence: Dr. Hasna Fahmima Haque, FCPS (Medicine), Registrar, Department of Medicine, BIRDEM General Hospital \& Ibrahim Medical College.

Received: 20 March 2014

Accepted: 20 April 2015 of adjacent structures or intracardiac (rarely extracardiac) shunting caused by rupture of the SVA. We report an unusual case of ruptured sinus of Valsalva aneurysm associated with infective endocarditis.

\section{Case Report:}

A 24-year-old male presented at Medicine out-patient department (OPD) of BIRDEM General Hospital with exertional dyspnoea, palpitation and mild central chest pain for seven days; patient also had a 2 months history of intermittent fever, episodic palpitation, loss of appetite and $2 \mathrm{~kg}$ of weight loss without any cough and syncope. His history was negative for drug abuse and dental procedures and tuberculosis work up was unremarkable. Prior to visiting our OPD, he was treated with complete courses of several broad-spectrum antibiotics without any improvement. On examination the patient was febrile $\left(103^{\circ} \mathrm{F}\right)$, he had tachycardia (heart rate 110 beats/minute) with bounding pulse, BP was 110/50 mmHg and his fingers and toes were clubbed; abdominal examination revealed mild splenomegaly. Cardiac apex was shifted to $11 \mathrm{~cm}$ from the midline at left $6^{\text {th }}$ Inter-costal space (ICS) and was thrusting; there was thrill as well as a continuous machinery murmur over the left $2^{\text {nd }}$ to $4^{\text {th }}$ ICS at the sternal edge. Other systemic examinations including fundoscopy were insignificant. Laboratory investigations showed neutrophilic leucocytosis (WBC $12000 / \mathrm{cmm}$, Neutrophil - 80\%), high ESR (75 mm in $1^{\text {st }}$ 
hour) and raised C-reactive protein (CRP) $(20 \mathrm{mg} / \mathrm{ml})$; urine R/E was normal and blood culture (FAN method) showed no growth. His ECG showed sinus tachycardia, chest X-ray (PA) view showed mild increase of the cardiothoracic index and transthoracic two- dimensional echocardiogram revealed rupture of the aneurysm of right sinus of valsalva with a large vegetation attached to the margin of the rupture-orifice (Fig -1) and shunting of blood from aortic root to right atrium in colour-doppler image (Fig-2). Ultrasonogram of whole abdomen showed splenomegaly. He was kept in absolute bed rest, treated with antipyretics, low dose diuretics and was given intravenous Ceftriaxone and Gentamicin for 4 weeks \& 2 weeks respectively. After 4 weeks, he became afebrile, his Complete Blood Count (CBC) \& $\mathrm{CRP}$ were normal and vegetation became smaller in size. In the meantime cardiothoracic surgical consultation was taken for repairing of the ruptured SVA and surgery was deferred as the patient's condition was improving with antibiotics.

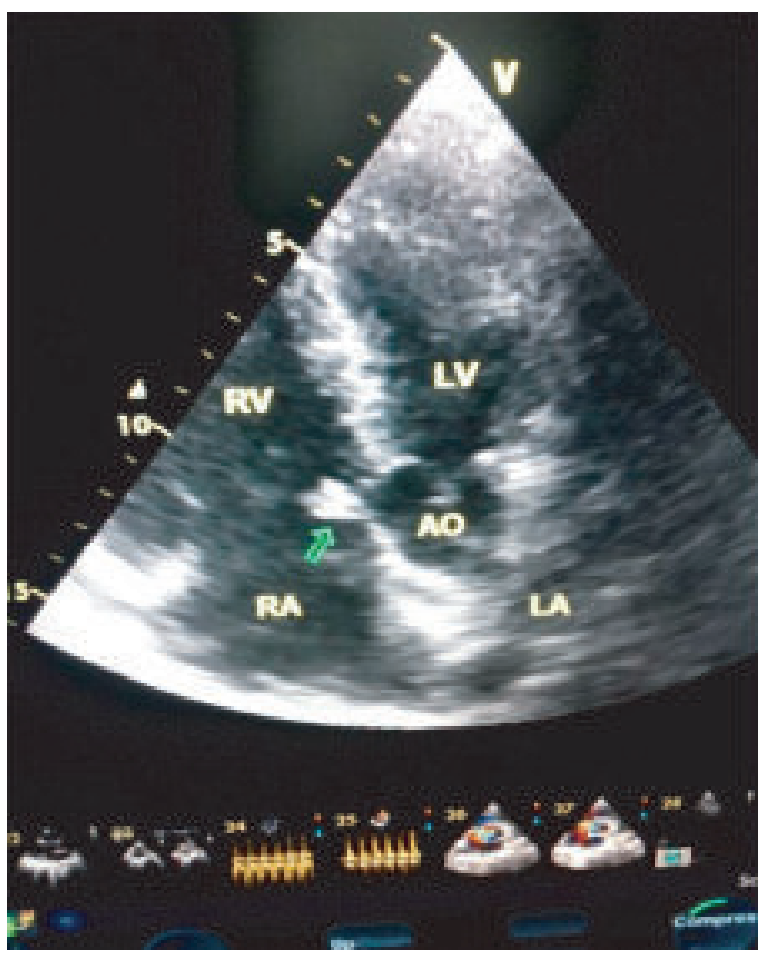

Fig-1: Transthoracic two - dimensional echocardiogram showing vegetation attached to the margin of the 'rupture-orifice' of right sinus of valsalva aneurysm.

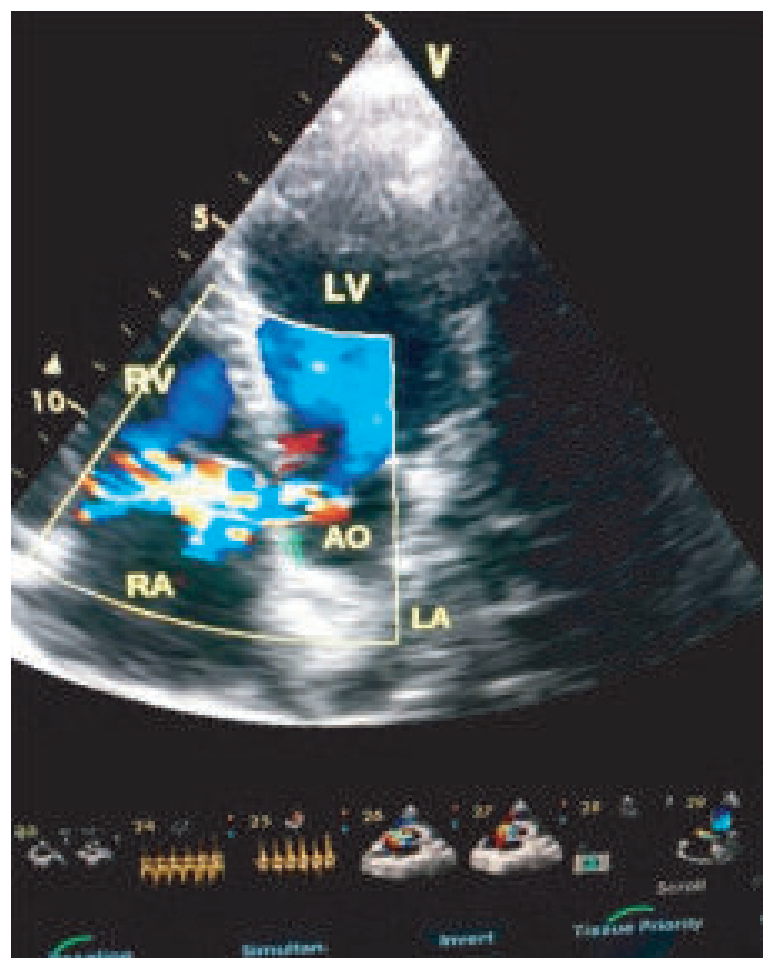

Fig-2: Colour - Doppler echocardiogram showing shunting of blood from aortic root to right atrium through ruptured right sinus of valsalva.

\section{Discussion:}

John Thurnam first described SVA in 1840; Hope further described it in 1939. SVA can be congenital or acquired. Congenital SVAs are caused by separation or failed fusion of the aortic media with the fibrosus annulus of the aortic valve. ${ }^{5}$ It comprises up to $3.5 \%$ of all congenital cardiac anomalies \& is more prevalent in males and people from Asian descent. Coexisting cardiac lesions, especially a ventricular septal defect or aortic valve regurgitation, may both be present in about 30 to $40 \%$ of patients. ${ }^{6-8}$ Approximately $65-85 \%$ of SVA originate from the right sinus of Valsalva, while SVAs originating from noncoronary $(10-30 \%)$ and left sinuses $(<5 \%)$ are exceedingly rare. ${ }^{9}$

A congenital SVA is usually clinically silent but may vary from a mild, asymptomatic dilatation detected incidentally during cardiac catheterization or echocardiography to symptomatic presentations related to the compression of adjacent structures or intracardiac (rarely extracardiac) shunting caused by rupture of the SVA. ${ }^{10}$ Unruptured SVA may lead to a compression of an adjacent chamber, a coronary artery or the conduction 
system, leading to myocardial ischaemia and/or conduction disturbances. Symptoms occur in $80 \%$ of patients, most commonly between 30 and 45 years of age. ${ }^{3}$ An aneurysm of the sinus of Valsalva ruptures in $35 \%$, leading to acute symptoms in one fourth of the patients. ${ }^{11}$ Rupture of a SVA occurs principally at the right ventricle (RV) $60 \%$ or at the right atrium (RA) $29 \%$, the left atrium (LA) $6 \%$ or the left ventricle (LV) $4 \%$. Extracardiac ruptures are rare, usually fatal, and occur towards the pericardium (1\%); leading to tamponade or the pleural space, pulmonary artery or superior caval vein and are more common when the SVA is of acquired origin. ${ }^{12}$ Symptoms associated with rupture are shortness of breath, chest pain, cough and fatigue. ${ }^{13,14}$ The severity of the shunt, the presence of associated lesions, and age at presentation determine the severity of symptoms. ${ }^{3}$ The most characteristic sign of rupture of SVA is a loud, superficial, "machine-type" continuous murmur that is usually accentuated in diastole associated with or without a superficial continuous thrill. The location of the murmur is along the right or left lower parasternal border when rupture occurs in RA, along mid to lower left parasternal border when rupture occurs in RV body and along upper left parasternal border when rupture occurs in RV outflow tract; other precordial findings are forceful and LV type apex beat and a brisk parasternal heave due to RV volume overload. Other clinical signs are bounding (high volume) or water hammer peripheral pulses and significantly increased pulse pressure on occasion. Patients may present with features of congestive cardiac failure and arrhythmias. ${ }^{15}$ In our case the patient's long clinical history of fever, anorexia, weight loss, \& palpitation together with clubbed fingers \& toes, mild splenomegaly $\&$ high fever at presentation and negative work up for tuberculosis raised strong suspicion of sub-acute infective endocarditis. His presenting complaints of exertional dyspnoea, palpitation \& central chest pain along with the continuous machinery murmur over the left sternal edge with associated thrill and thrusting \& shifted apex beat almost certainly diagnosed ruptured SVA on the background of sub-acute infective endocrditis.

Our patient had neutrophilic leukocytosis and high ESR as well as raised CRP in blood picture and splenomegaly on USG of W/A; these findings were consistent with bacterial endocarditis. Patient's negative blood culture report was probably due to several courses of broad spectrum antibiotics in recent past.
ECG findings in the cases of ruptured SVA are ventricular hypertrophy, electrical axis towards the right and atrial fibrillation. Transthoracic echocardiogram (TTE) and transesophageal echocardiogram (TEE) have a diagnostic accuracy of $75 \%$ and $90 \%$, respectively, for ruptured or unruptured SVA and allow accurate evaluation of the involved sinus, severity and direction of a shunt when ruptured, presence of associated cardiac or vascular abnormalities as well as the orientation of the surgical procedure. ${ }^{12,16}$ Magnetic resonance imaging (MRI) and Computed Tomography (CT) offer high diagnostic effectiveness. ${ }^{12}$ Cardiac catheterization is currently only performed to evaluate coronary anatomy prior to surgery. ${ }^{16}$ In our patient the ECG showed sinus tachycardia. TTE revealed ruptured sinus of valsalva aneurysm with shunting of blood from aortic root to RA with vegetation (Fig-1 \& 2). TEE, cardiac MRI and cardiac catheterization had not been done as the patient's condition improved and were reserved to do prior to surgery.

The optimal management for a ruptured SVA is surgical repair. The mean survival in patients with an untreated ruptured SVA is about four years. ${ }^{11}$ The most used surgical technique is the 'dual exposure technique' where both the aorta and chamber of termination are explored. The aneurysmal sac is excised and the resultant defect is repaired either by direct suturing or patch closure. Coexisting lesions, if present, are repaired within the same surgical procedure. To date, the ten-year survival rate after surgical repair of a ruptured SVA is $90 \%{ }^{4}$ However, aortic regurgitation might occur after surgical repair. ${ }^{2,11}$ Our patient was managed conservatively for bacterial endocarditis and later referred to cardiothoracic surgeon for repair of ruptured sinus of Valsalva.

In conclusion, rupture of SVA is a rare complication of infective endocarditis. Sudden onset of dyspnoea \& chest pain in the presence of a continuous loud murmur might be due to a ruptured sinus of Valsalva. Echocardiography and magnetic resonance imaging are useful for diagnosis. Left untreated it carries a dire prognosis. The treatment of choice is surgerical repair with management of any underlying treatable cause like infective endocarditis. 


\section{References}

1. Murray EG, Minami K, Kortke H, Segewiss H, Korfer R. Traumatic sinus of Valsalva fistula and aortic valve rupture. Ann Thorac Surg 1993; 55: 760-61.

2. Shumacker Jr HB. Aneurysms of the aortic sinuses of Valsalva due to bacterial endocarditis, with special reference to their operative management. J Thorac Cardiovasc Surg 1972; 63: 896-902.

3. Smith WA. Aneurysm of the sinus of Valsalva, with report of 2 cases. J Am Med Assoc 1914; 62: 1878.

4. Koh KK, Lee KH, Kim SS, Lee SC, Jin SH, Cho SW. Ruptured aneurysm of the sinus of Valsalva in a patient with Behcet's disease. Int J Cardiol 1994; 47: 177-79.

5. Lakoumentas JA, Bonou MS, Brili S, Theocharis CS, Benroubis AD, Perpinia AS, et al. Ruptured Aneurysm of the Right Sinus of Valsalva into the Right Ventricle. Hellenic J Cardiol 2002; 43: 242-45.

6. Jung SH, Yun TJ, Im YM, Park JJ, Song H, Lee JW, et al. Ruptured sinus of Valsalva aneurysm: transaortic repair may cause sinus of Valsalva distortion and aortic regurgitation. J Thorac Cardiovasc Surg 2008; 135: 1153-58.

7. Moustafa S, Mookadam F, Cooper L, Adam G, Zehr K, Stulak J, et al. Sinus of Valsalva aneurysms -47 years of a single center experience and systematic overview of published reports. Am J Cardiol 2007; 99:1159-64.

8. Wang ZJ, Zou CW, Li DC, Li HX, Wang AB, Yuan GD, et al. Surgical repair of sinus of Valsalva aneurysm in Asian patients. Ann Thorac Surg 2007; 84:156-60.
9. Meier JH, Seward JB, Miller FA, Jr, Oh JK, EnriquezSarano M. Aneurysms in the left ventricular outflow tract: clinical presentation, causes, and echocardiographic features. J Am Soc Echocardiogr 1998; 11(7): 729-45.

10. Ring WS. Congenital Heart Surgery Nomenclature and Database Project: Aortic Aneurysm, Sinus of Valsalva Aneurysm, and Aortic Dissection. Ann Thorac Surg 2000; 69: 147-63.

11. Yan F, Huo Q, Qiao J, Murat V, Ma SF. Surgery for sinus of valsalva aneurysm: 27-year experience with 100 patients. Asian Cardiovasc Thorac Ann 2008; 16:361-65.

12. Galicia-Tornell MM, Solís BM, Astorga OM, Anguiano SE, Martínez MM, Mendoza EV. Sinus of Valsalva aneurysm with rupture. Case report and literature review. Cir Ciruj 2009; 77: 441-45.

13. Reichert CL. Ruptured sinus Valsalva aneurysm, a rare cause of heart failure. Neth Heart J 2008; 16: 60-61.

14. Weijerse A, van der Schoot MJ, Maat LP, Bruning TA, Geleijnse ML, Bogers AJ. Cardiac tamponade due to a ruptured aneurysm of the sinus of valsalva. J Card Surg 2008; 23: 256-58.

15. Kaul UA, Yusuf J. Aneurysms of sinuses of Valsalva. In: Satpathy M, Mishra BR, eds. Clinical diagnosis of congenital heart disease. New Delhi: Jaypee Brothers Medical Publishers (P) Ltd; 2008: 170-77.

16. Post MC, Braam RL, Groenemeijer BE, Nicastia D, Rensing BJ, Schepens MA. Rupture of right coronary sinus of Valsalva aneurysm into right ventricle. Netherlands Heart Journal 2010; 18(4): 209-11. 